\title{
Comparative bioavailability of two oral formulations of clopidogrel: Determination of clopidogrel and its carboxylic acid metabolite (SR26334) under fasting and fed conditions in healthy subjects
}

\author{
NINA BRVAR ${ }^{1, *}$ \\ SYLVAIN LACHANCE ${ }^{2}$ \\ ANN LÉVESQUE ${ }^{2}$ \\ MARJANCA BREZNIK ${ }^{1}$ \\ LEA CVITKOVIČ-MARIČIČ ${ }^{1}$ \\ MATEJA MERSLAVIČ ${ }^{1}$ \\ IZTOK GRABNAR ${ }^{3}$ \\ TATJANA MATEOVIĆ-ROJNIK ${ }^{1}$ \\ ${ }^{1}$ Krka, d.d., Novo mesto \\ 8501 Novo mesto, Slovenia \\ 2 inVentiv Health Clinical \\ Québec, Canada \\ 3 University of Ljubljana, \\ Faculty of Pharmacy, 1000 Ljubljana \\ Slovenia
}

Accepted October 2, 2013

\begin{abstract}
Two randomized, single dose, 2-period, 2-sequence crossover studies were conducted to evaluate the comparative bioavailability of two clopidogrel formulations under fasting and fed conditions. Assessment of bioequivalence was based upon measurement of plasma concentrations of the parent drug, clopidogrel, and its major (inactive) metabolite, clopidogrel carboxylic acid, using improved methanol-free extraction.

Bioequivalence of Krka's formulation to the innovator's formulation was demonstrated under both fasting and fed conditions on 205 volunteers. Confidence intervals for $A U C_{0-\mathrm{t}}, A U C_{0 \text {-inf }}$ and $C_{\max }$ of clopidogrel and its main metabolite were well within the acceptance range of 80.00 to $125.00 \%$. Food substantially increased the bioavailability of clopidogrel from both formulations, while no effect of food on the extent and rate of exposure to the metabolite was observed. The effect of food was comparable between the two formulations, as indicated by the same direction and rank of food impact on the bioavailability of both formulations.
\end{abstract}

Keywords: bioavailability, food effect, clopidogrel, clopidogrel carboxylic acid

Clopidogrel, a thienopyridine derivative, is a potent antiplatelet drug that inhibits adenosine diphosphate (ADP)-mediated platelet activation and aggregation by selectively and irreversibly binding to platelet $\mathrm{P}_{2} \mathrm{Y}_{12}$ purinergic receptor (1). Results of a large clinical trial have demonstrated an overall benefit of clopidogrel over aspirin in the prevention of vascular ischemic events (myocardial infarction, stroke, vascular death) in patients with a history of symptomatic atherosclerotic disease (2). However, platelet inhibition by clopidogrel is highly variable, and patients with reduced platelet inhibition have an increased risk of major cardiovascular events $(3,4)$.

\footnotetext{
* Correspondence; e-mail: nina.brvar@krka.biz
} 
N. Brvar et al.: Comparative bioavailability of two oral formulations of clopidogrel: Determination of clopidogrel and its carboxylic acid metabolite (SR26334) under fasting and fed conditions in healthy subjects, Acta Pharm. 64 (2014) 45-62.

To exhibit its antiaggregatory effect, clopidogrel has to undergo intestinal absorption and cytochrome P450-dependent hepatic metabolism to form an active thiol metabolite (5-7). However, only a small proportion of administered clopidogrel is metabolized by P450 (15\% of total metabolites). Most of clopidogrel is hydrolyzed by esterases to an inactive carboxylic acid metabolite (SR26334) that accounts for approximately $85 \%$ of the clopidogrel-related compounds circulating in plasma (5-8).

Food has been reported to affect the bioavailability of clopidogrel and/or its metabolites (9-11). McEwen and coworkers (9) have showed that administration of a single dose of clopidogrel with food has no effect on the extent of exposure to the primary inactive metabolite of clopidogrel (SR26334) (90\% CI for AUC ratio was 0.90-1.02 for the fed/fasting state) and concluded that net absorption of clopidogrel was not significantly modified by food. However, $C_{\max }$ was lower in the fed compared to the fasting state by about $21 \%$. In a separate study performed by Nirogi and coworkers (10), the effect of concomitant food intake on the bioavailability of a single oral dose of clopidogrel was examined by estimating the parent drug clopidogrel in plasma. The pharmacokinetic (PK) parameters were significantly affected by food intake. Specifically, $C_{\max }$ and $A U C$ increased 6.1-fold and 9.2-fold, respectively, under fed conditions compared to fasting conditions. Similar results have been obtained in a recent study performed by Hurbin et al. (11). As assessed by $A U C$ and $C_{\max }$, single dose administration of clopidogrel with food resulted in a 6.13-fold (90\% CI, 5.31-7.08) and 4.20-fold (90\% CI, 3.57-4.93) increase in clopidogrel exposure, respectively. In the multiple dose study, the corresponding values were 3.32-fold (90\% CI, 2.88-3.84) and 2.46-fold (90\% CI; 2.03-2.99).

Due to the substantial effect of food on the pharmacokinetics of clopidogrel, the presented studies were conducted in fasted and fed states. The objective of the studies was to compare the bioavailability of two oral formulations of clopidogrel, Clopidogrel 75 mg film-coated tablets (Krka, d.d.), as test formulation, and Plavix ${ }^{\circledR} 75 \mathrm{mg}$ film-coated tablets (Sanofi-Synthelabo Limited), as reference formulation, after single dose administration under both fasting and fed conditions. Assessment of bioequivalence was based upon measurement of plasma concentrations of the parent drug, clopidogrel, using improved methanol-free extraction from plasma and its major (inactive) metabolite, clopidogrel carboxylic acid. The improved methanol-free extraction is crucial for clopidogrel determination as it prevents any metabolite back-conversion into clopidogrel. The effect of food on the pharmacokinetics of the two formulations was also examined.

\section{EXPERIMENTAL}

\section{Study protocol}

Two separate studies were performed to assess the bioequivalence of test and reference products. Bioequivalence assessment was performed under both fasting (Study I) and fed conditions (Study II).

The clinical part of the fasting study (Study I) was conducted at the MDS Pharma Services Clinical Research Centre, Saint-Laurent, Montréal, Québec, Canada, and the clinical part of the fed study (Study II) was conducted at the inVentiv Health Clinical Clinical Research Facility, Québec City, Québec, Canada. 
N. Brvar et al: Comparative bioavailability of two oral formulations of clopidogrel: Determination of clopidogrel and its carboxylic acid metabolite (SR26334) under fasting and fed conditions in healthy subjects, Acta Pharm. 64 (2014) 45-62.

The studies were carried out in compliance with the guidelines of the International Committee on Harmonisation (ICH) on Good Clinical Practice (GCP) (12), local regulatory requirements, the ethical requirements of Directive 2001/20/EC(13) and Directive 2001/83/EC as amended by Directive 2003/63/EC (14) and the principles enunciated in the Declaration of Helsinki and its revisions (15).

The protocol and informed consent forms (ICFs) of both studies were reviewed and approved by the Ethics Committee (Institutional Review Board convening at 2350 Cohen Street, Saint-Laurent, Montréal, Québec, Canada, for the fasting study and at 372 Holland view Trail, Suite 300, Aurora, Ontario, Canada, for the fed study) prior to initiation of study procedures. Written informed consent was obtained from all subjects before they underwent any study-specific procedures.

\section{Study population}

Healthy, non-smoking adult (age 18-55 years) male volunteers with a body mass index (BMI) between 18 and $30 \mathrm{~kg} / \mathrm{m}^{2}$ were enrolled in the studies. All subjects met the inclusion criteria and no exclusion criteria described in the protocol and were judged eligible for enrolment in the studies based on medical and medication histories, demographic data (including gender, age, race, body weight, height and BMI), vital signs measurements (blood pressure, heart rate, respiratory rate and body temperature), 12-lead ECG, physical examination, urine drug screen, urine cotinine test, alcohol screen and clinical laboratory tests (hematology, biochemistry, urinalysis, human immunodeficiency virus (HIV) test, hepatitis C (HCV) antibodies, hepatitis B surface antigen (HBsAg)).

Subjects who had donated 50 to $499 \mathrm{~mL}$ of blood within 30 days or more than $499 \mathrm{~mL}$ within 56 days prior to the first dose or had participated in another clinical trial within 28 days prior to the first dose were excluded.

In addition, volunteers were instructed to abstain from food and drinks containing xanthine derivatives or xanthine-related compounds at least 24 hours before dosing and throughout the period of sample collection in each period; alcohol-based products from at least 24 hours prior to admission and until the end of sample collection in each period; food and beverages containing grapefruit products at least 7 days prior to the first dose and throughout the study; vitamins at least throughout the confinement period; medication (including-over-the-counter products) or herbal products at least 7 days prior to the first dose and throughout the study.

A total of 96 and 118 subjects were included in the fasting and fed studies, respectively.

\section{Study design}

Both trials were designed as open-label, randomized, single dose, 2-treatment, 2-period, 2-sequence crossover bioequivalence studies.

In each period of both studies, subjects were confined to the clinical site from at least 10 hour before to 28 hours after administration of the study drug.

In each study, subjects were randomly assigned to receive a single oral dose of either the test formulation (Clopidogrel 75-mg film-coated tablets containing $75 \mathrm{mg}$ of clopidogrel (as hydrochloride), manufactured by KRKA, d.d., Novo mesto, Slovenia) or the 
N. Brvar et al.: Comparative bioavailability of two oral formulations of clopidogrel: Determination of clopidogrel and its carboxylic acid metabolite (SR26334) under fasting and fed conditions in healthy subjects, Acta Pharm. 64 (2014) 45-62.

reference formulation (Plavix ${ }^{\circledR} 75$-mg film-coated tablets containing $75 \mathrm{mg}$ of clopidogrel (as hydrogensulphate), manufactured by Sanofi-Synthelabo Limited, United Kingdom) during the first period and the alternative formulation during the second period.

In the fasting study, there was a 14-day washout period between the doses. The study was dosed in two groups of 48 . The two groups were dosed 7 days apart at the same clinical site following the same protocol requirements and procedures. The fed study was dosed in one group with doses separated by a washout period of 7 days.

In both studies, study drugs were administered with $240 \mathrm{~mL}$ of water (room temperature). Mouth and hand checks were performed after administration of each dose to ensure that volunteers had ingested all the medication.

In the fasting study, food was restricted at least 10 hours before dosing and 4 hours post-dose. In the fed study, subjects received a standardized breakfast after a supervised overnight fast of at least 10 hours. Subjects were required to completely consume their breakfast within 30 minutes prior to drug administration.

In both studies, water was not permitted from 1 hour before dosing to 1 hour following dosing, but was allowed at all other times. Standard meals were provided at approximately 4 and 9 hours after dosing, and at appropriate times thereafter. Post-dose meals were identical for both periods.

During both studies, subjects were required to remain ambulatory or seated for the first 4 hours after dosing in each period.

\section{Sample collection and processing}

Blood samples $(6 \mathrm{~mL})$ were collected in blood collection tubes containing EDTA $\mathrm{K}_{2}$ before dosing and at the following times thereafter: $0.167,0.333,0.5,0.667,0.833,1,1.25$, $1.5,2,3,4,5,6,8,12,16,24$ and 28 hours for the fasting study and $0.250,0.500,0.750$, $1.00,1.25,1.50,1.75,2.00,2.25,2.50,2.75,3.00,3.50,4.00,5.00,6.00,7.00,8.00,12.0,16.0$, 24.0 and 28.0 hours for the fed study.

Samples were cooled in an ice bath immediately after collection and centrifuged under refrigeration $\left(1500 \mathrm{~g}, 10\right.$ minutes, $\left.4^{\circ} \mathrm{C}\right)$ within 110 minutes of collection. Plasma samples were divided into 4 aliquots and stored frozen at $-20 \pm 5^{\circ} \mathrm{C}$ within 180 minutes from the start of centrifugation until assayed in the laboratory of inVentiv Health Clinical, Canada.

\section{Determination of clopidogrel and clopidogrel carboxylic acid (SR 26334) in plasma}

Chemicals and materials. - Clopidogrel hydrogen sulphate and clopidogrel- ${ }^{2} \mathrm{H}_{3}$ hydrogen sulphate were purchased from SynFine Research Inc. (Ontario, Canada). Ammonium formate was bought from Fluka (Oakville, Canada). Sodium bicarbonate, formic acid, acetonitrile $(\mathrm{ACN})$, hexanes and ethyl acetate were obtained from EMD (Toronto, Canada). Human plasma was bought from Valley Biomedical (Winchester, USA).

Clopidogrel carboxylic acid hydrochloride was purchased from SynFine Research Inc. (Ontario, Canada) and ticlopidine hydrochloride was purchased from Sigma-Aldrich (Ontario, Canada). 
N. Brvar et al.: Comparative bioavailability of two oral formulations of clopidogrel: Determination of clopidogrel and its carboxylic acid metabolite (SR26334) under fasting and fed conditions in healthy subjects, Acta Pharm. 64 (2014) 45-62.

Calibration standards and quality control preparation. - Stock solutions of clopidogrel and clopidogrel- ${ }^{2} \mathrm{H}_{3}$ at a concentration of $100 \mu \mathrm{g} \mathrm{mL}-1$ and subsequent intermediate solutions were prepared in acetonitrile for the calibration and quality control sample preparation.

Clopidogrel carboxylic acid and ticlopidine stock solutions were prepared at a concentration of 1 and $0.5 \mathrm{mg} \mathrm{mL}^{-1}$ in methanol, respectively.

For the fasting study, clopidogrel calibration standards were prepared in human EDTA $K_{2}$ plasma to obtain final concentrations around 5, 10, 125, 250, 500, 1000, 2000 and $2500 \mathrm{pg} \mathrm{mL}^{-1}$. Quality control samples were prepared in human EDTA $\mathrm{K}_{2}$ plasma at final concentrations of $15,187.5,1250$ and $1875 \mathrm{pg} \mathrm{mL}^{-1}$. For the fed study, calibration standards were prepared around 7.5, 15, 150, 750, 1500, 3000, 6000 and $7500 \mathrm{pg} \mathrm{mL}^{-1}$ and quality control samples at final concentrations of 22.5, 375, 2625 and $5625 \mathrm{pg} \mathrm{mL}^{-1}$.

For clopidogrel carboxylic acid, the same analytical range was used for the fasting and fed studies. Clopidogrel carboxylic acid calibration standards were prepared in human EDTA K 2 plasma at final concentrations around 4, 8, 40, 400, 800, 1600, 2400, 3200 and $4000 \mathrm{ng} \mathrm{mL}^{-1}$. Quality controls were prepared at concentrations of 12, 600, 1200 and $2800 \mathrm{ng} \mathrm{mL}^{-1}$.

Extraction method. - Clopidogrel was extracted from human plasma by liquid-liquid extraction using a methanol-free method. Clopidogrel was extracted by adding $150 \mu \mathrm{L}$ of plasma to the internal standard working solution prepared in Milli-Q type water. $1 \mathrm{~mL}$ of sodium bicarbonate buffer $200 \mathrm{mmol} \mathrm{L}^{-1}$ was added to the mixture and mixed thoroughly. The extraction solvent, a mixture of hexane/ethyl acetate (85:15) was distributed to each tube. After shaking for 15 minutes and centrifugation for 5 minutes at $1500 \mathrm{~g}$ at $4{ }^{\circ} \mathrm{C}$ to separate the organic and aqueous phases, the top layer was transferred to a borosilicate tube and evaporated to dryness. The dry residue was then reconstituted with mobile phase composed of water/acetonitrile (25:75) and ammonium formate 5 $\mathrm{mmol} \mathrm{L}^{-1}$. The $\mathrm{pH}$ of the mobile phase was adjusted to 6.0 with formic acid.

The analytical method for the analyte clopidogrel carboxylic acid and its internal standard ticlopidine was protein precipitation with acetonitrile. Acetonitrile was added to the $0.050 \mathrm{~mL}$ aliquot of human EDTA plasma, mixed well and then centrifuged. A portion of the supernatant was diluted with the mobile phase (Milli-Q type water/methanol (55:45), $1 \mathrm{mmol} \mathrm{L}^{-1}$ ammonium formate) to be injected into the analytical column and mass spectrometer.

Chromatography and mass spectrometry conditions. - Reverse phase high performance liquid chromatography was chosen to achieve separation in both assays. An API 4000 triple quadrupole mass spectrometer ( $\mathrm{AB}$ Sciex, Concord, Ontario, Canada) with the Analyst 1.4.1 software was used for the detection and data acquisition of all experiments. Positive multiple reaction monitoring (MRM) mode with a TurboIonSpray source was selected. The HPLC components included a Hewlett Packard 1100 Series solvent delivery module and a Perkin Elmer Series 200 autosampler.

MRM transitions monitored were $322.2 \rightarrow 212.2$ amu and $327.2 \rightarrow 217.3$ amu for clopidogrel mobile phase was a mixture of Milli-Q type water $(25 \%)$, acetonitrile $(75 \%)$ and ammonium formate $5 \mathrm{mmol} \mathrm{L}^{-1}$ pumped at a flow rate of $1 \mathrm{~mL} \mathrm{~min}^{-1}$. The column used was a Zorbax SB-C8 $75 \times 4.6 \mathrm{~mm} 3.5 \mu \mathrm{m}$ at room temperature and the injection volume 
N. Brvar et al.: Comparative bioavailability of two oral formulations of clopidogrel: Determination of clopidogrel and its carboxylic acid metabolite (SR26334) under fasting and fed conditions in healthy subjects, Acta Pharm. 64 (2014) 45-62.

was $40 \mu \mathrm{L}$. Quantitation was obtained with a calibration curve using the peak area ratio (analyte/internal standard) and $1 / x^{2}$ weighted linear regression.

MRM transitions monitored were $308.1 \rightarrow 198.2$ amu and $264.2 \rightarrow 125.1$ amu for clopidogrel carboxylic acid and ticlopidine, respectively. Positive ionization using the TurboIonSpray ${ }^{\mathrm{TM}}$ was used for determination. The Collision Energy was set at 22 and $44 \mathrm{~V}$ for clopidogrel carboxylic acid and ticlopidine, respectively. The mobile phase was pumped at a flow rate of $1 \mathrm{~mL} \mathrm{~min}^{-1}$ and was a mixture of Milli-Q type water/methanol (55:45), 1 mmol L-1 ammonium formate. Separation was achieved on a Zorbax SB-C18, $4.6 \times 50$ $\mathrm{mm}, 5 \mu \mathrm{m}$ column at room temperature and the injection volume was $20 \mu \mathrm{L}$. The same type of quantitation as clopidogrel was used.

\section{Pharmacokinetic analyses}

The pharmacokinetic parameters were calculated for plasma clopidogrel and its carboxylic acid metabolite (SR26334).

$C_{\max }$, maximum observed concentration, and $T_{\max }$, time to reach $C_{\max }$, were determined directly from the plasma concentration data. $A U C_{0-t}$, area under the plasma concentration-time curve from time 0 to the time of the last measurable (non-zero) concentration was calculated using the linear trapezoidal rule. $A U C_{0 \text {-inf }}$, area under the plasma concentration-time curve from time 0 to infinity was calculated as $A U C_{0-\mathrm{t}}+C_{\mathrm{t}} / K_{\mathrm{el}}$, where $C_{\mathrm{t}}$ was the last non-zero concentration. $K_{\mathrm{el}}$, elimination rate constant was determined by linear regression of the terminal phase of the ln concentration-time data. $T_{1 / 2}$, elimination half-life, was calculated as $\ln 2 / K_{\mathrm{el}}$. Residual area (\%), the percentage of extrapolated area under the curve, was calculated as $\left(1-\left(A U C_{0-\mathrm{t}} / A U C_{0 \text {-inf }}\right)\right) \times 100$.

All pharmacokinetic analyses were performed using PhAST (software developed at MDS) and Bioequiv (software developed at inVentiv Health Clinical, Canada) for the fasting and fed studies, respectively.

\section{Statistical analyses}

For both clopidogrel and clopidogrel carboxylic acid, the analysis of variance (ANOVA) was carried out on the ln-transformed PK parameters $A U C_{0-\mathrm{t}}, A U C_{0 \text {-inf }}$ and $C_{\text {max }}$, using $S A S^{\circledR}$ GLM procedure (SAS ${ }^{\circledR}$ for Windows ${ }^{\mathrm{TM}}$, SAS Institute, Cary, NC, USA).

In the fasting study, which was performed in two groups, the ANOVA model included group, sequence, period nested within group, formulation, formulation* group interaction and subject nested within group*sequence effects. ANOVA model in the fed study, in which subjects were dosed in one group, included sequence, subject within sequence, period and treatment as factors.

Each ANOVA included calculations of least squares means (LSM), the difference between formulation LSM and the standard error associated with this difference. Consistent with the two one-sided tests for bioequivalence, $90 \%$ confidence intervals (CI) for the ratios were derived by exponentiation of the confidence intervals obtained for the difference between formulation LSM resulting from the analyses on the ln-transformed $A U C_{0-\mathrm{t}}, A U C_{0 \text {-inf }}$ and $C_{\text {max }}$. Confidence intervals were expressed as percentage relative to the reference formulation. Finally, the intra-subject variability (ISCV) for the $A U C_{0-t}$, $A U C_{0 \text {-inf }}$ and $C_{\max }$ parameters were also determined. 
N. Brvar et al.: Comparative bioavailability of two oral formulations of clopidogrel: Determination of clopidogrel and its carboxylic acid metabolite (SR26334) under fasting and fed conditions in healthy subjects, Acta Pharm. 64 (2014) 45-62.

Bioequivalence was assessed for clopidogrel as per standards of $90 \%$ geometric confidence interval for ln-transformed $A U C_{0-\mathrm{t}}, A U C_{0 \text {-inf }}$ and $C_{\max }$ within the 80.00-125.00\% interval. Results for clopidogrel carboxylic acid were considered as supportive data.

Furthermore, the impact of food on the pharmacokinetics of clopidogrel and clopidogrel carboxylic acid was also assessed for both formulations by comparing ln-transformed $A U C_{0-t}, A U C_{0-\text { inf }}$ and $C_{\text {max }}$ parameters under fed and fasting conditions using the two-sample $t$-test with Satterthwaite approximation. Geometric mean ratios were estimated and geometric $90 \%$ confidence intervals for ratios were calculated using fasted values as reference. Statistical evaluation was performed using the SAS ${ }^{\circledR}$ TTEST procedure.

\section{Safety evaluation}

Safety was assessed by the monitoring of adverse events (whether elicited by query, spontaneously reported or observed by the clinical staff) or determined by vital signs measurements and standard laboratory evaluations (hematology, urinalysis and serum chemistry). Adverse events were monitored throughout the study until resolution and were described in terms of severity, seriousness, outcome, action, frequency and relationship to treatments.

\section{RESULTS AND DISCUSSION}

\section{Study population}

Demographic summary, including age, race, height, body weight and BMI of populations from both studies, is presented in Table I.

In fasting and fed studies, all 96 and 118 subjects, respectively, received at least one dose of the study medication and comprised the safety population.

92 of the 96 subjects in study I (fasting conditions) and 113 of the 118 subjects in study II (fed conditions) completed both periods of the study. All subjects that completed the studies were included in the pharmacokinetic and statistical analyses, except for subject No. 50 of the fed study, who was excluded from the pharmacokinetic and statistical analyses of clopidogrel because of a clot at $1.50 \mathrm{~h}$ in period 1 that affected the subject's $T_{\max }$ and $C_{\max }$. Therefore, the number of subjects included in the analyses was 92 in the fasting study and 112 and 113 for clopidogrel and clopidogrel carboxylic acid, respectively, in the fed study.

\section{Determination of clopidogrel and clopidogrel carboxylic acid (SR 26334) in plasma}

Assessment of the back-conversion of a metabolite in the presence of methanol. - During preliminary experiments, involving samples of a previous study performed by inVentiv Health Clinical, in which the original method involving methanol in extraction was used, irreproducibility of the clopidogrel concentrations determined was observed when the samples were re-assayed. It was deemed necessary to investigate these discrepancies. The areas investigated pointed to the following conclusions. 
N. Brvar et al.: Comparative bioavailability of two oral formulations of clopidogrel: Determination of clopidogrel and its carboxylic acid metabolite (SR26334) under fasting and fed conditions in healthy subjects, Acta Pharm. 64 (2014) 45-62.

Table I. Summary of demographic characteristics of the study populations for Study I (fasting conditions) and Study II (fed conditions)

\begin{tabular}{|c|c|c|}
\hline Category & Study I (fasting conditions) & Study II (fed conditions) \\
\hline$N$ & 96 & 118 \\
\hline \multicolumn{3}{|l|}{ Age (years) } \\
\hline Mean \pm SD & $36 \pm 8$ & $33 \pm 9$ \\
\hline Range & 18 to 52 & 18 to 54 \\
\hline \multicolumn{3}{|l|}{ Race } \\
\hline Asian & $1(1.0 \%)$ & 0 \\
\hline Black & $6(6.3 \%)$ & $2(1.7 \%)$ \\
\hline Caucasian & $89(92.7 \%)$ & $116(98.3 \%)$ \\
\hline Other & 0 & 0 \\
\hline \multicolumn{3}{|l|}{ Height $(\mathrm{cm})$} \\
\hline Mean \pm SD & $174 \pm 6-174.2 \pm 6.4$ & $175 \pm 7$ \\
\hline Range & 161 to 191 & 162 to 196 \\
\hline \multicolumn{3}{|l|}{ Mass (kg) } \\
\hline Mean \pm SD & $75.6 \pm 7.0$ & $81.2 \pm 8.3$ \\
\hline Range & 60.3 to 89.7 & 63.7 to 99.1 \\
\hline \multicolumn{3}{|l|}{$B M I\left(\mathrm{~kg} / \mathrm{m}^{2}\right)$} \\
\hline Mean \pm SD & $24.9 \pm 1.8$ & $26.4 \pm 2.3$ \\
\hline Range & 20.7 to 27.8 & 21.5 to 29.9 \\
\hline
\end{tabular}

In the original method, clopidogrel was extracted from plasma by liquid-liquid extraction. Internal standard stock solutions were prepared in methanol, while the working internal standard solution was prepared in a methanol/water mixture (50:50). Plasma was added to the internal standard working solution and the extraction buffer (sodium bicarbonate $\mathrm{pH}$ 9.5). The proportion of methanol in this mixture was $3.87 \%$. As methanol involved in the sample extraction procedure was suspected to cause overestimation of clopidogrel concentrations in study samples, a series of experiments were conducted with incurred samples from a previous clinical study. Since the metabolite involved in back-conversion was unknown, it was more adequate to use real clinical samples for the experiments. The following experiments using different percentages of methanol and acetonitrile in internal standard solutions were performed to demonstrate the impact of methanol on the metabolite back-conversion:

A. Internal standard stock solution in methanol, internal standard intermediate solution in methanol and internal standard working solution in water/methanol (50:50) like 
N. Brvar et al:: Comparative bioavailability of two oral formulations of clopidogrel: Determination of clopidogrel and its carboxylic acid metabolite (SR26334) under fasting and fed conditions in healthy subjects, Acta Pharm. 64 (2014) 45-62.

in the original method for a proportion of methanol of $3.87 \%$ in the plasma, internal standard working solution and buffer solution mixture.

B. Internal standard stock solution in methanol, internal standard intermediate solution in methanol and internal standard working solution in water for a proportion of methanol of $0.04 \%$ in the plasma, internal standard working solution and buffer solution mixture.

C. Internal standard stock solution in methanol, internal standard intermediate solution in methanol and internal standard working solution in water/acetonitrile (50:50) for a proportion of methanol of $0.04 \%$ in the plasma, internal standard working solution and buffer solution mixture.

D. Internal standard stock solution in methanol, internal standard intermediate solution in acetonitrile and internal standard working solution in water/acetonitrile (50/50) for a proportion of methanol of $0.0025 \%$ in the plasma, internal standard working solution and buffer solution mixture.

These solutions were added to the plasma samples and the extraction buffer (sodium bicarbonate at $\mathrm{pH}$ 9.5) and the resulting mixtures were kept at room temperature for 0 and 30 minutes prior to extraction and analysis. Results are presented in Table II.

The presence of methanol in the sample extraction procedure enhanced the back-conversion of one of clopidogrel metabolites. Overestimation of clopidogrel concentrations increased proportionally with methanol quantity in the extraction process. Moreover, degradation of this metabolite increased with the extraction time as demonstrated in Table II. It was found that a low proportion (0.04 \%) of methanol induced an increase of approximately $5 \%$ of clopidogrel concentration in 30 minutes (experiments $\mathrm{B}, \mathrm{C}$ ). However, $0.04 \%$ of methanol induced an increase of clopidogrel concentrations up to 15 $\%$ when compared to the experiment with only $0.0025 \%$ of methanol (experiments B, C vs. experiment D), showing that the amount of methanol must be at a minimum during the extraction. The improved methanol-free extraction is therefore the method of choice for clopidogrel measurement in human plasma.

Validation of the method. - Methanol-free extraction for the determination of clopidogrel in human plasma was thus validated in accordance with the Food and Drug Administration (FDA) validation guidelines (16). Different parameters were assessed during the validation: accuracy and precision, selectivity, sensitivity, analyte and internal standard recoveries, dilution integrity, matrix effect, hemolysis and lipemic effect, stabilities in matrix and in solution.

In quality control samples, clopidogrel was found to be stable in human EDTA $\mathrm{K}_{2}$ plasma for 739 days at $-20^{\circ} \mathrm{C}$, for 23 hours at room temperature and after four freeze-thaw cycles. Clopidogrel carboxylic acid was found to be stable for 252 days at $-20^{\circ} \mathrm{C}$, for 26 hours at room temperature and after four freeze-thaw cycles.

In addition, during method validation, clopidogrel stability during sample treatment (prior to the addition of extraction solvent) was evaluated. A pool of real clinical samples was incubated at room temperature with the internal standard solution prepared in Milli-Q type water (from an intermediate solution prepared in acetonitrile) and the extraction buffer (sodium bicarbonate $\mathrm{pH}$ 9.5) for 30 and 60 minutes. Results are presented in Table III. 
N. Brvar et al.: Comparative bioavailability of two oral formulations of clopidogrel: Determination of clopidogrel and its carboxylic acid metabolite (SR26334) under fasting and fed conditions in healthy subjects, Acta Pharm. 64 (2014) 45-62.

Table II. Change in clopidogrel concentration due to back-conversion of a metabolite vs. methanol content in sample extraction

\begin{tabular}{cccccc}
\hline Experiment & $\begin{array}{c}\text { Ratio of } \\
\text { methanol in } \\
\text { extraction (\%) }\end{array}$ & $\begin{array}{c}\text { Incubation } \\
\text { time (min) }\end{array}$ & $\begin{array}{c}\text { Clopidogrel } \\
\text { conc. } \\
\left(\mathrm{pg} \mathrm{mL}^{-1}\right)\end{array}$ & $\begin{array}{c}\text { Change vs. } \\
\text { time }=0 \\
(\%)\end{array}$ & $\begin{array}{c}\text { Change vs. solution } \\
\text { with 0.0025\% of } \\
\text { methanol }(\%)\end{array}$ \\
\hline A & 3.87 & 0 & 950.55 & & 366.5 \\
\hline B & 0.04 & 0 & 1327.53 & 39.7 & 551.5 \\
\hline C & 0.04 & 0 & 222.68 & & 9.3 \\
& & 30 & 235.07 & 5.6 & 15.4 \\
\hline D & 0.0025 & 0 & 204.53 & & 0.4 \\
& & 30 & 214.72 & 5.0 & 5.4 \\
\hline
\end{tabular}

Experiment A, working internal standard (IS) solution in $\mathrm{H}_{2} \mathrm{O} / \mathrm{MeOH}$ (50:50) and IS intermediate solution in $\mathrm{MeOH}$; Experiment B, working IS solution in $\mathrm{H}_{2} \mathrm{O}$ and IS intermediate solution in $\mathrm{MeOH}$; Experiment $\mathrm{C}$, working IS solution in $\mathrm{H}_{2} \mathrm{O} / \mathrm{ACN}$ (50:50) and IS intermediate solution in $\mathrm{MeOH}$; Experiment D, working IS solution $\mathrm{H}_{2} \mathrm{O} / \mathrm{ACN}(50: 50)$ and IS intermediate solution in CAN.

It was shown that the assay was highly reproducible when done without methanol. No changes in clopidogrel concentrations were observed when substituting methanol with acetonitrile and water in the solutions used prior to study sample extraction while maintaining all the other extraction steps and reagents unchanged. This indicates that there was no back-conversion of clopidogrel metabolites with the improved methanol-free extraction.

\section{Pharmacokinetic evaluation}

Mean clopidogrel and clopidogrel carboxylic acid concentration-time profiles are presented in Figs. 1 and 2 for the fasting and fed conditions, respectively. Table IV summarizes descriptive statistics for the main pharmacokinetic parameters of clopidogrel

Table III. Impact of incubation time at room temperature during sample extraction on clopidogrel using improved methanol-free extraction

\begin{tabular}{cccc}
\hline & \multicolumn{3}{c}{ Clopidogrel concentration $\left(\mathrm{pg} \mathrm{mL}^{-1}\right)$} \\
\cline { 2 - 4 } & 0 & Incubation time at r.t. $(\mathrm{min})$ & 60 \\
\hline Average & 110.16 & 30 & 109.21 \\
$\mathrm{SD}$ & 1.830 & 3.089 & 0.170 \\
$\mathrm{CV}(\%)$ & 1.7 & 2.8 & 0.2 \\
Difference $(\%)$ & $\mathrm{N} / \mathrm{Ap}$ & -0.02 & -0.85 \\
\hline
\end{tabular}


N. Brvar et al: Comparative bioavailability of two oral formulations of clopidogrel: Determination of clopidogrel and its carboxylic acid metabolite (SR26334) under fasting and fed conditions in healthy subjects, Acta Pharm. 64 (2014) 45-62.

and clopidogrel carboxylic acid, obtained for the two tested formulations under both fasting and fed conditions.

As seen from Figs. 1 and 2, the profiles of test and reference formulations show similar absorption, distribution and elimination under both fasting and fed conditions.

After administration of both test and reference formulations under fasting conditions, clopidogrel was rapidly absorbed, with median $T_{\max }$ of clopidogrel and carboxylic acid metabolite occurring within 1 hour after dosing. Mean $A U C_{0-\mathrm{t}}, A U C_{0 \text {-inf }}$ and $C_{\max }$ of clopidogrel were 2257.62, 2579.18 and $1106.70 \mathrm{pg} \mathrm{mL}^{-1}$, respectively, for the test formulation and 1909.67, 1983.37 and $981.37 \mathrm{pg} \mathrm{mL}^{-1}$, respectively, for the reference formulation. Mean $A U C_{0-\mathrm{t}}, A U C_{0 \text {-inf }}$ and $C_{\text {max }}$ of clopidogrel carboxylic acid for the respective formulations were $6632.39 \mathrm{ngh} \mathrm{mL}^{-1}, 7105.75 \mathrm{ngh} \mathrm{mL}^{-1}, 2545.68 \mathrm{ng} \mathrm{mL}^{-1}$ and 6774.74 ngh $\mathrm{mL}^{-1}, 7229.61 \mathrm{ngh} \mathrm{mL}^{-1}$ and $2608.86 \mathrm{ng} \mathrm{mL}^{-1}$.

In the presence of food, absorption was somewhat delayed for both analytes, as indicated by median $T_{\max }$ of clopidogrel and carboxylic acid metabolite occurring 1.75 hours after administration of the test formulation and 1.50 hours after administration of the reference formulation. Food substantially increased the rate and extent of clopidogrel absorption; mean values for $A U C_{0-\mathrm{t}}, A U C_{0 \text {-inf }}$ and $C_{\max }$ of clopidogrel were 5784.53 pg h mL-1, $5880.63 \mathrm{pg} \mathrm{h} \mathrm{mL}^{-1}$ and $3176.73 \mathrm{pg} \mathrm{mL}^{-1}$, respectively, for the test formulation and $5546.89 \mathrm{pg} \mathrm{h} \mathrm{mL}^{-1}, 5631.20 \mathrm{pg} \mathrm{h} \mathrm{mL}^{-1}$ and $3002.99 \mathrm{pg} \mathrm{mL}^{-1}$, respectively, for the reference formulation. Mean $A U C_{0-\mathrm{t}}, A U C_{0 \text {-inf }}$ and $C_{\text {max }}$ of clopidogrel carboxylic acid for the respective formulations were $6513.08 \mathrm{ng} \mathrm{h} \mathrm{mL}^{-1}, 6845.59 \mathrm{ng} \mathrm{h} \mathrm{mL}^{-1}, 2284.18 \mathrm{ng} \mathrm{mL}^{-1}$ and $6510.43 \mathrm{ng} \mathrm{h} \mathrm{mL}^{-1}, 6836.09 \mathrm{ng} \mathrm{h} \mathrm{mL}^{-1}$ and $2193.73 \mathrm{ng} \mathrm{mL}^{-1}$.

The design of the studies was adequate for determining the pharmacokinetic parameters of the test and reference formulations.
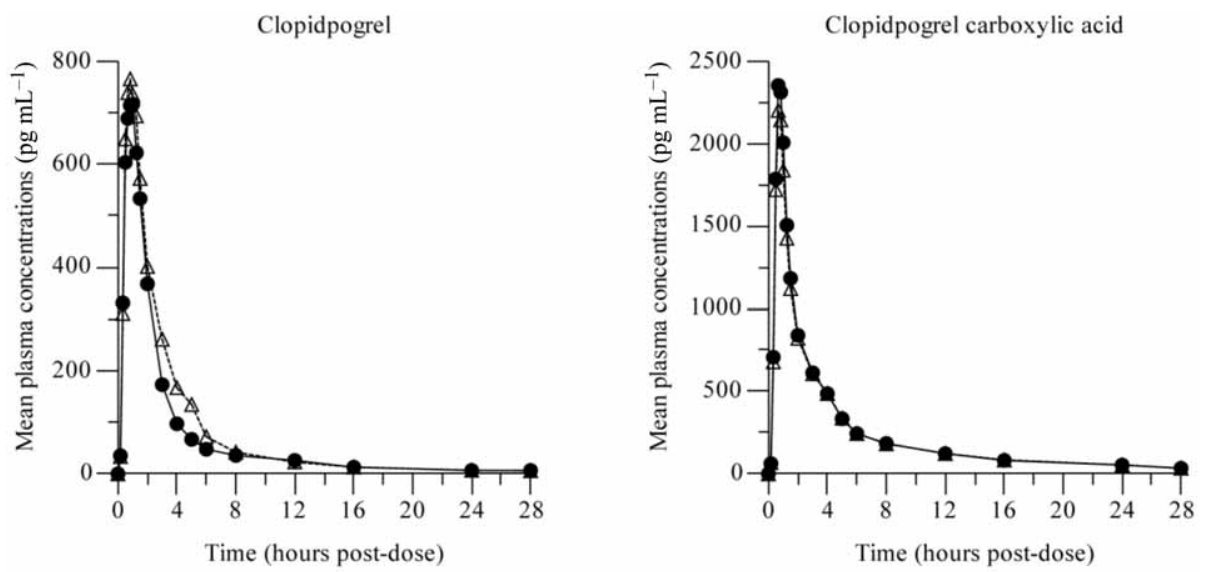

$\rightarrow-$ Reference (fasting) $\quad \cdots$ - Test (fasting)

Fig. 1. Mean plasma concentration-time profiles of clopidogrel (left panel; $n=92$ ) and clopidogrel carboxylic acid (right panel; $n=92$ ) following single-dose administration of test and reference formulations of clopidogrel $75 \mathrm{mg}$ tablets in healthy subjects under fasting conditions (data from Study I). 
N. Brvar et al.: Comparative bioavailability of two oral formulations of clopidogrel: Determination of clopidogrel and its carboxylic acid metabolite (SR26334) under fasting and fed conditions in healthy subjects, Acta Pharm. 64 (2014) 45-62.

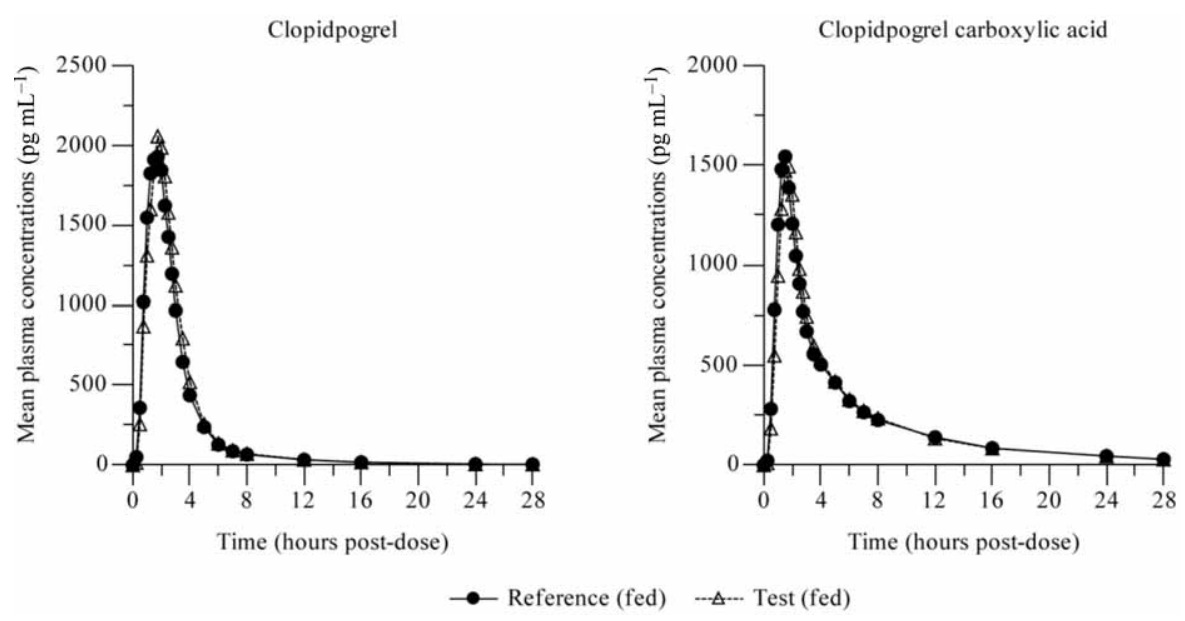

Fig. 2. Mean plasma concentration-time profiles of clopidogrel (left panel; $n=112$ ) and clopidogrel carboxylic acid (right panel; $n=113$ ) following single-dose administration of test and reference formulations of clopidogrel $75 \mathrm{mg}$ tablets in healthy subjects under fed conditions (data from Study II).

In both studies, the selection and timing of blood samples were judged appropriate to adequately characterize the pharmacokinetics of clopidogrel and carboxylic acid metabolite since there was no subject with $C_{\max }$ being the first point of the concentration-time curve and the mean extrapolated portion of the $A U C_{0 \text {-inf }}$ of clopidogrel and clopidogrel carboxylic acid was very low for the test and reference products under both tested conditions. Finally, there was no detectable concentration of clopidogrel and carboxylic acid metabolite in the pre-dose samples of period 2 in either study, suggesting that the washout periods in both studies were adequate to allow complete elimination of the drug before period 2 and to avoid any carryover.

\section{Statistical evaluation}

The least squares means ratios as well as the $90 \%$ geometric confidence intervals for the ratios of $\ln$-transformed $A U C_{0-\mathrm{t}}, A U C_{0 \text {-inf }}$ and $C_{\max }$ of the test to reference products are presented in Table V.

In both studies, the confidence intervals for $A U C_{0-\mathrm{t}}, A U C_{0 \text {-inf }}$ and $C_{\max }$ of clopidogrel were well within the acceptance range of 80.00 to $125.00 \%$, indicating that the test formulation was bioequivalent to the reference formulation under both fasting and fed conditions. Although in terms of the demonstration of bioequivalence, the parent compound, clopidogrel, is essential, results of clopidogrel carboxylic acid, although therapeutically inactive, indicate that also metabolic processes occur at a comparable rate and extent in test and reference formulations, which gives further evidence of equivalence in in vivo performance.

Intra-subject variability for clopidogrel PK parameters $A U C_{0-\mathrm{t}}, A U C_{0 \text {-inf }}$ and $C_{\max }$ was $43.29,43.52$ and $54.63 \%$, respectively, under fasting conditions and 23.78, 23.52 and 
N. Brvar et al.: Comparative bioavailability of two oral formulations of clopidogrel: Determination of clopidogrel and its carboxylic acid metabolite (SR26334) under fasting and fed conditions in healthy subjects, Acta Pharm. 64 (2014) 45-62.

Table IV. Pharmacokinetic parameters of clopidogrel and clopidogrel carboxylic acid following single-dose administration of test and reference formulations of clopidogrel $75 \mathrm{mg}$ tablets in healthy subjects under fasting (Study I) and fed (Study II) conditions

\begin{tabular}{|c|c|c|c|c|}
\hline \multirow[t]{2}{*}{ Parameters $^{\mathrm{a}}$} & \multicolumn{2}{|c|}{$\begin{array}{l}\text { Study I (fasting conditions) } \\
\text { ( } n=92 \text { for clopidogrel and } \\
\text { clopidogrel carboxylic acid) }\end{array}$} & \multicolumn{2}{|c|}{$\begin{array}{c}\text { Study II (fed conditions) } \\
\left(n=112^{c} \text { for clopidogrel and } n=113\right. \\
\text { for clopidogrel carboxylic acid) }\end{array}$} \\
\hline & Test & Reference & Test & Reference \\
\hline \multicolumn{5}{|c|}{ Clopidogrel } \\
\hline 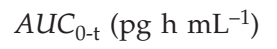 & $2257.62(3482.86)$ & 1909.67 (1575.74) & $5784.53(2675.80)$ & $5546.89(2704.29)$ \\
\hline 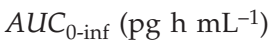 & $2579.18(3891.35)^{d}$ & $1983.37(1649.49)^{e}$ & $5880.63(2743.19)^{f}$ & $5631.20(2753.74)^{f}$ \\
\hline Residual area $(\%)$ & $6.63(7.55)^{\mathrm{d}}$ & $6.46(6.36)^{\mathrm{e}}$ & $1.73(0.91)^{\mathrm{f}}$ & $1.75(0.93)^{\mathrm{f}}$ \\
\hline$C_{\max }\left(p g \mathrm{~mL}^{-1}\right)$ & $1106.70(1557.67)$ & 981.37 (1000.96) & 3176.73 (1691.29) & 3002.99 (1832.68) \\
\hline$T_{\max }(\mathrm{h})^{\mathrm{b}}$ & $\begin{array}{c}1.00 \\
(0.333-16.00)\end{array}$ & $\begin{array}{c}1.00 \\
(0.333-5.00)\end{array}$ & $\begin{array}{c}1.75 \\
(0.50-3.50)\end{array}$ & $\begin{array}{c}1.50 \\
(0.50-5.00)\end{array}$ \\
\hline$K_{\mathrm{el}}\left(\mathrm{h}^{-1}\right)$ & $0.1398(0.0806)^{\mathrm{d}}$ & $0.1289(0.0539)^{\mathrm{e}}$ & $0.1475(0.0543)^{\mathrm{f}}$ & $0.1475(0.0515)^{\mathrm{f}}$ \\
\hline$T_{1 / 2}(\mathrm{~h})$ & $7.11(6.24)^{\mathrm{d}}$ & $6.90(4.74)^{\mathrm{e}}$ & $5.50(2.33)^{\mathrm{f}}$ & $5.39(2.12)^{\mathrm{f}}$ \\
\hline
\end{tabular}

Clopidogrel carboxylic acid

\begin{tabular}{ccccc}
\hline$A U C_{0-\mathrm{t}}\left(\mathrm{ng} \mathrm{h} \mathrm{mL} \mathrm{mL}^{-1}\right)$ & $6632.39(1372.74)$ & $6774.74(1435.91)$ & $6513.08(1201.22)$ & $6510.43(1194.87)$ \\
$\left.A U C_{0 \text {-inf }}(\mathrm{ng} \mathrm{h} \mathrm{mL})^{-1}\right)$ & $7105.75(1452.37)$ & $7229.61(1536.30)$ & $6845.59(1327.37)$ & $6836.09(1267.02)$ \\
Residual area (\%) & $6.64(3.98)$ & $6.26(3.24)$ & $4.69(1.99)$ & $4.71(2.07)$ \\
$\left.C_{\max }(\mathrm{ng} \mathrm{mL})^{-1}\right)$ & $2545.68(873.25)$ & $2608.86(854.89)$ & $2284.18(625.60)$ & $2193.73(655.20)$ \\
$T_{\max }(\mathrm{h})^{\mathrm{b}}$ & 0.685 & 0.775 & 1.75 & 1.50 \\
$K_{\mathrm{el}}\left(\mathrm{h}^{-1}\right)$ & $0.0816(0.0201)$ & $0.0821(0.0188)$ & $0.0953(0.0158)$ & $0.0964(0.0167)$ \\
$T_{1 / 2}(\mathrm{~h})$ & $9.06(2.46)$ & $8.89(2.08)$ & $7.49(1.32)$ & $7.43(1.46)$ \\
\hline
\end{tabular}

a Arithmetic mean (standard deviation)

b Median (minimum-maximum)

c Subject No. 50 was excluded from the pharmacokinetic and statistical analyses of clopidogrel because of a clot at $1.50 \mathrm{~h}$ in period 1 that affected $T_{\max }$ of the subject.

d-f $A U C_{0 \text {-inf }}$, Residual area, $K_{\mathrm{el}}$ and $T_{1 / 2}$ could not be calculated for some subjects. For these parameters, $\mathrm{d} n=83$, e $n=86,{ }^{\mathrm{f}} n=111$.

$32.93 \%$, respectively, under fed conditions. The corresponding values for clopidogrel carboxylic acid were $8.36,7.83$ and $26.03 \%$ for the fasting conditions and 5.74, 5.79 and $19.68 \%$ for the fed conditions. High intra-subject variability of clopidogrel pharmacokinetic parameters, observed in both studies, confirms the adequate design of the two studies also with regard to the number of subjects included in the studies. Calculated intra-subject variability was reasonably low for clopidogrel carboxylic acid. 
N. Brvar et al.: Comparative bioavailability of two oral formulations of clopidogrel: Determination of clopidogrel and its carboxylic acid metabolite (SR26334) under fasting and fed conditions in healthy subjects, Acta Pharm. 64 (2014) 45-62.

Clopidogrel pharmacokinetics varied widely also among subjects. Coefficients of variation of $C_{\max }$ and $A U C$ of clopidogrel ranged from 82.51 to $154.27 \%$ in the fasting state and from 46.26 to $61.03 \%$ in the fed state. Like intra-individual variability, inter-individual variability of clopidogrel carboxylic acid was lower; the corresponding ranges were 20.44 to $34.30 \%$ and 18.35 to $29.87 \%$.

Highly variable pharmacokinetics, observed in our clopidogrel studies, are consistent with literature data $(4,17)$ and have been explained by several factors, including demographic factors (age and body mass) (4), variability in intestinal drug absorption (due to polymorphism in the transporter molecule P-glycoprotein, commonly referred to as ATP-binding cassette protein B1, ABCB1) (17-21) and cytochrome P450-dependent enzyme activity (CYP3A4 activity, genetic polymorphism in CYP2C19) (21-24). Likely contributors to this variation in clopidogrel pharmacokinetics include still unidentified factors (genetic factors, other than CYP2C19 and P-glycoprotein polymorphism, and nongenetic sources of variation in clopidogrel absorption and metabolism) (4).

Food substantially increased clopidogrel bioavailability from both test and reference formulations. Specifically, $A U C_{0-\mathrm{t}}, A U C_{0 \text {-inf }}$ and $C_{\max }$ increased 3.80-fold (90\% CI 3.19, $4.51)$, 3.32-fold (90 \% CI 2.78, 3.97) and 4.71-fold (90\% CI 3.84, 5.78), respectively, for the test formulation, and 3.63-fold ( $90 \%$ CI 3.09, 4.25), 3.48-fold (90 \% CI 2.97, 4.07) and 4.13-fold $(90 \%$ CI 3.43, 4.99), respectively, for the reference formulation.

As regards inactive metabolite, food had practically no effect on the extent and rate of exposure, as indicated by fasting/fed ratios for $A U C_{0-\mathrm{t}}, A U C_{0 \text {-inf }}$ and $C_{\max } 0.99(90 \%$ CI $0.94,1.03), 0.97$ (90\% CI 0.92, 1.01) and 0.93 (90\% CI 0.85, 1.00), respectively, for the

Table V. Summary of the results of statistical analyses of clopidogrel and clopidogrel carboxylic acid comparing the test formulation with the reference formulation under fasting (Study I) and fed conditions (Study II)

\begin{tabular}{|c|c|c|c|c|}
\hline \multirow[t]{2}{*}{ Parameters } & \multicolumn{2}{|c|}{$\begin{array}{l}\text { Study I (fasting conditions) } \\
\qquad(n=92)\end{array}$} & \multicolumn{2}{|c|}{$\begin{array}{c}\text { Study II (fed conditions) } \\
(n=112 \text { for clopidogrel and } n=113 \\
\text { for clopidogrel carboxylic acid) }\end{array}$} \\
\hline & Ratio of LSM & $90 \%$ geometric CI & Ratio of LSM & $90 \%$ geometric CI \\
\hline \multicolumn{5}{|c|}{ Clopidogrel } \\
\hline$A U C_{0-\mathrm{t}}$ & $99.71 \%$ & $90.08-110.37 \%$ & $104.40 \%$ & $99.11-109.97 \%$ \\
\hline$A U C_{0 \text {-inf }}$ & $97.62 \%{ }^{a}$ & $87.35-109.09 \%$ a & $104.41 \% \mathrm{~b}$ & $99.15-109.94 \%$ b \\
\hline$C_{\max }$ & $95.00 \%$ & $83.82-107.67 \%$ & $108.22 \%$ & $100.79-116.20 \%$ \\
\hline \multicolumn{5}{|c|}{ Clopidogrel carboxylic acid } \\
\hline$A U C_{0-\mathrm{t}}$ & $97.98 \%$ & $96.00-100.01 \%$ & $100.00 \%$ & $98.74-101.27 \%$ \\
\hline$A U C_{0 \text {-inf }}$ & $98.41 \%$ & 96.55-100.32 \% & $99.99 \%$ & $98.72-101.27 \%$ \\
\hline$C_{\max }$ & $97.31 \%$ & 91.39-103.61 \% & $104.79 \%$ & $100.38-109.39 \%$ \\
\hline
\end{tabular}

a $n=86$

$\mathrm{b}_{n}=111$ 
N. Brvar et al.: Comparative bioavailability of two oral formulations of clopidogrel: Determination of clopidogrel and its carboxylic acid metabolite (SR26334) under fasting and fed conditions in healthy subjects, Acta Pharm. 64 (2014) 45-62.

test formulation, and 0.97 (90\% CI 0.92, 1.01), 0.95 (90\% CI 0.91, 1.00) and $0.86(90 \%$ CI $0.79,0.93)$, respectively, for the reference formulation.

Several factors that may account for the observed effect of food on clopidogrel pharmacokinetics have been reported $(11,25)$. In the Question \& Answers document issued by the Committee for Human Medicinal Products (25), the increase in clopidogrel bioavailability, observed under fed conditions, was hypothesized to be caused by reduced acidic hydrolysis in the stomach due to limited dissolution of clopidogrel in gastric media under fed conditions compared to extensive hydrolysis and formation of the inactive carboxylic acid metabolite under fasting conditions, which reduced the amount of unchanged clopidogrel available for absorption at the intestinal level. However, recent tests of in vitro dissolution tests performed by Hurbin and coworkers (11) showed that clopidogrel was not significantly degraded in a test solution of pH 2.0 (similar to fasted gastric $\mathrm{pH}$ ), with no evidence of acidic hydrolysis compared to solutions of $\mathrm{pH} 4.5$ and 6.8. Furthermore, the authors explained the significant increase in unchanged clopidogrel observed after concomitant administration of clopidogrel with food by a combination of food effects at the intestinal level (higher clopidogrel solubility and/or reduced precipitation) and decreased hepatic first-pass metabolism due to increased blood flow in the fed state. Clopidogrel is a high extraction ratio drug (11) and, hence, the increase in hepatic blood flow in the fed state is expected to decrease hepatic first-pass metabolism and increase clopidogrel bioavailability.

The effect of food was comparable between the two formulations, as indicated by the same direction and rank of food impact on the bioavailability (AUC and $C_{\max }$ ) of both formulations. Furthermore, the effect of food on the pharmacokinetics of clopidogrel and its carboxylic acid metabolite, as observed in our studies, is consistent with that previously reported for clopidogrel and its inactive metabolite (9-11), where a significant effect of food on clopidogrel bioavailability ( $A U C$ and $C_{\max }$ ) was reported $(10,11)$. However, there was no food effect on the exposure to the primary metabolite of clopidogrel (SR 26334) (9).

The absence of a substantial food effect observed in our study as well as in the literature (9) for the inactive clopidogrel carboxylic acid is presumably related to the opposing effects of the increased liver blood flow (decreased first pass metabolism to carboxylic acid) and increased solubility and/or decreased precipitation of clopidogrel at the intestinal level (increased fraction of clopidogrel absorbed) under fed conditions.

\section{Safety and tolerability}

Adverse events (AEs) were reported in 17 of the 96 subjects and 42 of 118 subjects in the fasting and fed studies, respectively, who received at least one dose of the study medication (safety population). The most common AEs in the fasting study were headache (8 subjects; $8.3 \%$ ) and dizziness (5 subjects; $5.2 \%$ ); the most common AEs in the fed study were headache (13 subjects; $11.0 \%$ ), somnolence (5 subjects; $4.2 \%$ ) and dizziness (5 subjects; $4.2 \%$ ). No serious or severe AEs occurred during the two studies.

Of all AEs reported, 5 subjects $(5.3 \%$ of the study population receiving test formulation, altogether 11 AEs) experienced at least 1 adverse event that was possibly, probably, or definitely related to treatment, and 4 subjects $(4.3 \%$ of the study population receiving reference formulation, altogether 8 AEs) experienced at least 1 adverse event 
N. Brvar et al:: Comparative bioavailability of two oral formulations of clopidogrel: Determination of clopidogrel and its carboxylic acid metabolite (SR26334) under fasting and fed conditions in healthy subjects, Acta Pharm. 64 (2014) 45-62.

that was possibly, probably, or definitely related to treatment in the fasting study. In the fed study, 13 subjects ( $11.2 \%$ of the study population receiving test formulation, altogether $14 \mathrm{AEs})$ and 10 subjects $(8.7 \%$ of the study population receiving reference formulation, altogether $10 \mathrm{AEs}$ ) experienced at least 1 adverse event that was possibly, probably, or definitely related to treatment.

To summarize, test and reference formulations were well tolerated under both fasting and fed conditions, with no serious adverse events. No relevant differences in safety profiles were observed between the two formulations.

\section{CONCLUSIONS}

Platelet inhibition by clopidogrel is highly variable. A significant component of the inter-patient differences in responses to clopidogrel is undoubtedly its highly variable pharmacokinetics. Considering the pronounced food effect and the mentioned high variability in clopidogrel pharmacokinetics, which has not been fully explained yet, as well as its substantial inter-patient variability in response, associated with increased risk for cardiovascular events, additional in vivo testing under fed conditions can provide a significant contribution in terms of demonstration of comparable safety and efficacy of the two formulations.

\section{REFERENCES}

1. P. Savi, C. Labouret, N. Delesque, F. Guette, J. Lupker and J. M. Herbert, P2Y ${ }_{12}$, a new platelet ADP receptor, target of clopidogrel, Biochem. Biophys. Res. Commun. 283 (2001) 379-383; DOI: 10.1006/bbrc.2001.4816.

2. CAPRIE Steering Committee, A randomized, blinded, trial of clopidogrel versus aspirin in patients at risk of ischaemic events (CAPRIE), Lancet 348 (1996) 1329-1339; DOI: 10.1016/ S0140-6736(96)09457-3. Available online 10 January 2000.

3. L. Bonello, U. S. Tantry, R. Marcucci, R. Blindt, D. J. Angiolillo, R. Becker, D. L. Bhatt, M. Cattaneo, J. P. Collet, T. Cuisset, C. Gachet, G. Montalescot, L. K. Jennings, D. Kereiakes, D. Sibbing, D. Trenk, J. W. Van Werkum, F. Paganelli, M. J. Price, R. Waksman and P. A. Gurbel, Consensus and future directions on the definition of high on-treatment platelet reactivity to adenosine diphosphate, J. Am. Coll. Cardiol. 56 (2010) 919-933; DOI: 10.1016/j.jacc.2010.04.047.

4. A. L. Frelinger III, D. L. Bhatt, R. D. Lee, D. J. Mulford, J. Wu , S. Nudurupati, A. Nigam, M. Lampa, J. K. Brooks, M. R. Barnard and A. D. Michelson, Clopidogrel pharmacokinetics and pharmacodynamics vary widely despite exclusion or control of polymorphisms (CYP2C19, $\mathrm{ABCB} 1, \mathrm{PON1}$ ), noncompliance, diet, smoking, co-medications (including proton pump inhibitors), and pre-existent variability in platelet function, J. Am. Coll. Cardiol. 26 (2013) 872-879; DOI: $10.1016 /$ j.jacc.2012.11.040.

5. P. Savi, J. M. Pereillo, M. F. Uzabiaga, J. Combalbert, C. Picard, J. P. Maffrand, M. Pascal and J. M. Herbert, Identification and biological activity of the active metabolite of clopidogrel, Thromb. Haemost. 84 (2000) 891-986.

6. T. A. Clarke and L. A. Waskell, The metabolism of clopidogrel is catalyzed by human cytochrome P450 3A and is inhibited by atorvastatin, Drug Metab. Dispos. 31 (2003) 53-59; DOI: 10.1124/ dmd.31.1.53. 
N. Brvar et al.: Comparative bioavailability of two oral formulations of clopidogrel: Determination of clopidogrel and its carboxylic acid metabolite (SR26334) under fasting and fed conditions in healthy subjects, Acta Pharm. 64 (2014) 45-62.

7. P. Savi, J. Combalbert, C. Gaich, M. C. Rouchon, J. P. Maffrand, Y. Berger and J. M. Herbert, The antiaggregating activity of clopidogrel is due to a metabolic activation by the hepatic cytochrome P450-1A, Thromb. Haemost. 72 (1994) 313-317.

8. H. Caplain, F. Donat, C. Gaud and J. Necciari, Pharmacokinetics of clopidogrel, Semin. Thromb. Hemost. 25 (1999) 25-28.

9. J. McEwen, G. Strauch, P. Perles, G. Pritchard, T. E. Moreland, J. Necciari and J. P. Dickinson, Clopidogrel bioavailability: absence of influence of food or antacids, Semin. Thromb. Hemost. 25 (1999) 47-50.

10. R. V. Nirogi, V. N. Kandikere and K. Mudigonda, Effect of food on bioavailability of a single oral dose of clopidogrel in healthy male subjects, Arzneimittelforsch. 56 (2006) 735-739; DOI: $10.1055 /$ s-0031-1296783.

11. F. Hurbin, X. Boulenc, N. Daskalakis, C. Farenc, T. Taylor, D. Bonneau, F. Lacreta, S. Cheng and E. Sultan, Clopidogrel pharmacodynamics and pharmacokinetics in the fed and fasted state: a randomized crossover study of healthy men, Clin. Pharmacol. 52 (2012) 1506-1515; DOI: 10.1177 /0091270011419852.

12. European Medicines Agency, Committee for Proprietary Medicinal Products (CPMP), ICH Topic E6 (R1), Guideline for good clinical practice, Step 5, Note for guidance on good clinical practice,CPMP/ICH/135/95, July 2002; http://www.ema.europa.eu/ema/index.jsp?curl=pages / regulation/general/general_content_000429.jsp\&mid=WC0b01ac0580029590; access date April 22, 2013.

13. Directive 2001/20/EC of the European Parliament and of the Council of 4 April 2001 on the approximation of the laws, regulations and administrative provisions of the Member States relating to the implementation of good clinical practice in the conduct of clinical trials on medicinal products for human use, Off. J. Eur. Communities L Legis. L121 (2001) 34-44; http:/ /ec.europa.eu/ health/documents/eudralex/vol-1/; access date April 22, 2013.

14. Commission directive 2003/63/EC of 25 June 2003 amending Directive 2001/83/EC of the European Parliament and of the Council on the Community code relating to medicinal products for human use. Off. J. Eur. Communities L Legis. L159 (2003) 46-94; http://ec.europa.eu/health/ documents/eudralex/vol-1/; access date April 22, 2013.

15. World Medical Association Declaration of Helsinki, Ethical Principles for Medical Research Involving Human Subjects, 59th WMA General Assembly, October 2008; http:/ /www.wma.net/ en/30publications/10policies/b3/; access date April 22, 2013.

16. U.S. Department of Health and Human Services, Food and Drug Administration, Center for Drug Evaluation and Research (CDER), Center for Veterinary Medicine (CVM). Guidance for Industry, Bioanalytical Method Validation, May 2001; www.fda.gov/downloads/Drugs/ GuidanceComplianceRegulatoryInformation/Guidances/UCM070107.pdf; access date April 22, 2013.

17. D. Taubert, A. Kastrati, S. Harlfinger, O. Gorchakova, A. Lazar, N. von Beckerath, A. Schömig and E. Schömig, Pharmacokinetics of clopidogrel after administration of a high loading dose, Thromb. Haemost. 92 (2004) 311-316; DOI: 10.1160/TH04-02-0105.

18. N. von Beckerath, D. Taubert, G. Pogatsa-Murray, E. Schömig, A. Kastrati and A. Schömig, Absorption, metabolization, and antiplatelet effects of 300-, 600-, and 900-mg loading doses of clopidogrel, results of the ISAR-CHOICE (Intracoronary Stenting and Antithrombotic Regimen: Choose Between 3 High Oral Doses for Immediate Clopidogrel Effect) Trial, Circulation 112 (2005) 2946-2950; DOI: 10.1161/?CIRCULATIONAHA.105.559088.

19. D. Taubert, N. von Beckerath, G. Grimberg, A. Lazar, N. Jung, T. Goeser, A. Kastrati, A. Schömig and E. Schömig, Impact of P-glycoprotein on clopidogrel absorption, Clin. Pharmacol. Ther. 80 (2006) 486-501; DOI: 10.1016/j.clpt.2006.07.007.

20. A. R. Shuldiner, J. R.O'Connell, K. P. Bliden, A. Gandhi, K. Ryan, R. B. Horenstein, C. M. Damcott, R. Pakyz, U. S. Tantry, Q. Gibson, T. I. Pollin, W. Post, A. Parsa, B. D. Mitchell, N. Faraday, 
N. Brvar et al.: Comparative bioavailability of two oral formulations of clopidogrel: Determination of clopidogrel and its carboxylic acid metabolite (SR26334) under fasting and fed conditions in healthy subjects, Acta Pharm. 64 (2014) 45-62.

W. Herzog and P. A. Gurbel, Association of cytochrome P450 2C19 genotype with the antiplatelet effect and clinical efficacy of clopidogrel therapy, JAMA. 26 (2009) 849-857; DOI: 10.1001/ jama.2009.1232.

21. J. L. Mega, S. L. Close, S. D. Wiviott, L. Shen, J. R. Walker, T. Simon, E. M. Antman, E. Braunwald and M. S. Sabatine, Genetic variants in ABCB1 and CYP2C19 and cardiovascular outcomes after treatment with clopidogrel and prasugrel in the TRITON-TIMI 38 trial: a pharmacogenetic analysis, Lancet 376 (2010) 1312-1319; DOI: 10.1016/S0140-6736(10)61273-1.

22. T. Simon, C. Verstuyft, M. Mary-Krause, L. Quteineh, E. Drouet, N. Méneveau, P. G. Steg, J. Ferrières, N. Danchin and L. Becquemont, Genetic determinants of response to clopidogrel and cardiovascular events, N. Engl. J. Med. 360 (2009) 363-375; DOI: 10.1056/NEJMoa0808227.

23. W. C. Lau, L. A. Waskell, P. B. Watkins, C. J. Neer, K. Horowitz, A. S. Hopp, A. R. Tait, D. G. Carville, K. E. Guyer and E. R. Bates, Atorvastatin reduces the ability of clopidogrel to inhibit platelet aggregation: a new drug-drug interaction, Circulation 107 (2003) 32-37; DOI: 10.1161/ ?01.CIR.0000047060.60595.CC.

24. W. C. Lau, P. A. Gurbel, P. B. Watkins, C. J. Neer, A. S. Hopp, D. G. Carville, K. E. Guyer, A. R. Tait and E. R. Bates, Contribution of hepatic cytochrome P450 3A4 metabolic activity to the phenomenon of clopidogrel resistance, Circulation 109 (2004) 166-171; DOI: 10.1161/01.CIR. 0000112378.09325.F9.

25. European Medicines Agency, Committee for Human Medicinal Products (CHMP), Questions \& Answers: Positions on specific questions addressed to the pharmacokinetics working party, EMA/618604/2008 Rev. 7, February 2013; http://www.ema.europa.eu/ema/index.jsp?curl= pages/regulation/general/general_content_000370.jsp\&mid=WC0b01ac0580032ec5; access date April 22, 2013. 